\title{
On the puzzle of space weathering alteration of basaltic asteroids
}

\author{
S. Marchi \\ Department of Astronomy, Padova University \\ Vicolo dell'Osservatorio 3, I-35122 Padova, Italy \\ simone.marchi@unipd.it \\ M. C. De Sanctis \\ Istituto di Astrofisica Spaziale e Fisica Cosmica, INAF \\ Via Fosso del Cavaliere 100, I-00133 Roma, Italy \\ mariacristina.desanctis@iasf-roma.inaf.it \\ M. Lazzarin \\ Department of Astronomy, Padova University \\ Vicolo dell'Osservatorio 3, I-35122 Padova, Italy \\ monica.lazzarin@unipd.it \\ S. Magrin \\ Department of Astronomy, Padova University \\ Vicolo dell'Osservatorio 3, I-35122 Padova, Italy \\ sara.magrin.1@unipd.it
}

\begin{abstract}
The majority of basaltic asteroids are found in the inner main belt, although a few have also been observed in the outer main belt and near-Earth space. These asteroids -referred to as V-types- have surface compositions that resemble that of the $530 \mathrm{~km}$ sized asteroid Vesta. Besides the compositional similarity, dynamical evidence also links many V-type asteroids to Vesta. Moreover, Vesta is one of the few asteroids to have been identified as source of specific classes of meteorites, the howardite, eucrite, diogenite achondrites (HEDs).

Despite the general consensus on the outlined scenario, several questions remain unresolved. In particular, it is not clear if the observed spectral diversity among Vesta, V-types and HEDs is due to space weathering, as is thought to be the case for S-type asteroids.

In this paper, SDSS photometry is used to address the question of whether the spectral diversity among candidate V-types and HEDs can be explained by space weathering. We show that visible spectral slopes of V-types are systematically redder with respect to HEDs, in a similar way to what is found for ordinary chondrite meteorites and S-types. On the assumption that space weathering is responsible for the slope mismatch, we estimated an upper limit for the reddening timescale of about $0.5 \mathrm{Ga}$. Nevertheless, the observed slope mismatch between HEDs and Vtypes poses several puzzles to understanding its origin. The implication of our findings is also discussed in the light of Dawn mission to Vesta.
\end{abstract}

Subject headings: Minor planets, asteroids: general - minor planets, asteroids: individual (Vesta) meteorites, meteors, meteoroids 


\section{Introduction}

Vesta is the largest basaltic fully differentiated asteroid. Remote visible and near-infrared spectroscopy indicates the presence of basaltic mineralogy on Vesta's surface and the possible presence of other components (McCord et al. 1970; Larson \& Fink 1975; Gaffev 1997). A large number of small asteroids that show a similar surface composition, the so-called V-type asteroids, populates the inner main belt (Binzel \& Xu 1993; Duffard et al. 2004). V-type asteroids have dimensions less than a few tens of $\mathrm{km}$ that cannot sustain differentiation processes, therefore they must have been originated from one -or moremuch larger, differentiated parent body. Thus, it has been suggested that Vesta is the parent body for V-types (McCord et al. 1970).

To support this scenario, apart from the aforementioned compositional similarity, it has been shown that many V-types and Vesta belong to a large asteroid family (Marzari et al. 1996; Nesvorný et al. 2008). To reinforce this link, a $460 \mathrm{~km}$ wide crater has been detected on Vesta (Thomas et al. 1997). Another interesting aspect concerning Vesta and V-types is that their visible and near-infrared spectra have been found to be similar to those of a particular suite of meteorites, namely the howardite, eucrite and diogenite achondrites (HEDs; McCord et al. 1970; Consolmagno \& Drake 1977; Takeda 1997; Drake 2001).

On the other hand, it is also clear that neither all V-types nor HEDs are compatible with being derived from Vesta; therefore, it is suspected that they originate from multiple differentiated parent bodies (Yamaguchi et al. 2002; Wiechert et al. 2004). In this respect, it is significant that a few V-types have been discovered beyond the 3:1 mean motion resonance (Lazzaro et al. 2000; Duffard \& Roig 2009) which cannot be related to Vesta on dynamical basis Roig et al. 2008; Nesvorný et al. 2008) and -at least in the case of Magnya- also on compositional basis (Hardersen et al. 2004).

Despite the general scenario described above is largely accepted, there are some observations that remain unexplained. First of all, V-types generally exhibit stronger absorption bands than Vesta and steeper continuum both in the visible (Hiroi \& Pieters 1998) and in the 0.4-1.6 $\mu \mathrm{m}$ region (Burbine et al. 2001).
The reason for these discrepancies is not yet clear. Possible causes are indicated in composition/texture differences or due to the effects of some alteration process (e.g. Burbine et al. 2001), like for instance those that have been demonstrated to cause optical alteration on silicate-rich S-type asteroids (Jedicke et al. 2004; Marchi et al. 2006a). However, on S-types the 1 and $2 \mu \mathrm{m}$ absorption bands tend to become shallower by increasing space weathering (i.e. increasing spectral slope), while this effect is not observed on $\mathrm{V}$ types. V-type asteroids that have reddest slopes also show strong 1 and $2 \mu \mathrm{m}$ absorption bands (e.g. Burbine et al. 2001). Vesta is found to be most comparable to howardites with a fine-grained size distribution (e.g. Pieters et al. 2006).

On the other hand, the laboratory experiments conducted on HEDs and pyroxenes confirm that V-type materials should alter under the effects of ion bombardment and micrometeorite impacts, in a way similar to S-types, although the alteration timescale is likely somehow reduced (Hiroi \& Pieters 1998; Marchi et al. 2005a; Vernazza et al. 2006).

In this paper we present a comprehensive model of space weathering of candidate V-types detected via SDSS photometry (Roig \& Gil-Hutton 2006). The present analysis aims to investigate whether or not V-type surfaces are affected by space weathering processes. We identify several interesting facts that support the presence of space weathering alteration, although its behavior appears distinct to what found on S-types.

In doing so, our findings disclose interesting aspects of surface properties of Vesta and V-types that are useful also in the context of the NASA Dawn mission (Russell et al. 2007) for what concerns mission planning and Vesta's data interpretation.

\section{Modeling the space weathering of V- type asteroids}

\section{Spectrophotometric catalog of V-types}

It has been demonstrated for S-type asteroids that visible spectral slopes can be successfully used to model the optical alterations of asteroidal surfaces due to space weathering. Spectral slopes have been evaluated using different methods, mainly photometric data (e.g. Jedicke et al. 2004) and 
spectroscopy (Marchi et al. 2006a; Paolicchi et al. 2007), leading basically to the same conclusions. Concerning V-types, spectroscopic data are available only for a restricted number $(\sim 70)$ of objects, preventing any significant characterization of the whole population. For this reason, in the present work, we will make use of SDSS photometry reported in the Moving Object Catalog V3 (MOCV3; Ivezić et al. 2001; Jurić et al. 2002), which allows the analysis to be extended to include a large number of objects (388 and 466 family and non-family Vtypes, respectively). V-types have been selected according to Roig \& Gil-Hutton (2006). In addition, we also include $52 \mathrm{~V}$-types identified by the spectroscopic surveys SMASS, S3OS2, and SINEO (Lazzaro et al. 2004; Bus \& Binzel 2002; Binzel et al. 2004; Marchi et al. 2005b) which are not included in the SDSS MOCV3. It is important to underline that the list established by Roig \& Gil-Hutton (2006) consists of candidate V-types and that spectroscopic data are needed for a robust identification. Nevertheless, a comparison between candidate V-types and confirmed ones displays a good match (Roig \& Gil-Hutton 2006).

Spectral slopes have been evaluated by linear best fit of the albedo using the SDSS bands g', r',i', z'. One sigma errors of SDSS albedoes have been used as weighting factors for the fitting procedure. As for spectroscopic data, we first evaluated the albedo in g', r', i', z' bands through cubic spline of the spectra and then proceeded with linear best fit. Albedoes have been normalized to the r' band (Roig \& Gil-Hutton 2006).

\section{Parameterizing the space weathering}

Previous analysis of S-types and ordinary chrondrites (OCs) showed that the space weathering on asteroids is a process that, if no other alteration is present, is expected to progressively evolve over time until eventually a sort of saturation is reached. Moreover, its efficiency depends on the location and past orbital evolution of the asteroids.

In this work, the study of space weathering is done according to the model developed in a series of papers by Marchi et al. (2006a, b) and Paolicchi et al. (2007). This model demonstrated that the aging, location, and past evolution can be condensed into a single parameter, named exposure $E$. The exposure is proportional to the dose of radiation/ion received from the Sun and scales as

$$
E=\frac{T}{a^{2} \sqrt{1-e^{2}}}
$$

where $a, e$ are the Keplerian elements of the body, and $T$ is its average collisional age (Marchi et al. 2006a). For MBAs we used proper elements given their stability on a longer timescale. Ages are derived according to the collisional evolution of main belt asteroids Bottke et al. 2005; Marchi et al. 2006a). Collisional lifetimes depend upon the diameters, which have been estimated using the measured average geometric albedo (0.3) of a sample of V-types (Tedesco et al. 2002; Benner et al. 2002; Delbó et al. 2003, 2006). The collisional age of V-types spans from about 0.5 to $2.7 \mathrm{Ga}$. These extremes correspond to the smallest and largest bodies of the sample, which have estimated diameters of about $1 \mathrm{~km}$ and $10 \mathrm{~km}$, respectively. In what follows, it is assumed that the formation of Vesta's family occurred sometime before the oldest age in the sample, namely $\sim 2.7 \mathrm{Ga}$, so that asteroid's age are set by subsequent collisional evolution. Although Vesta's family age is not well constrained, a recent work of Nesvorný et al. (2008) found evidence for a much earlier origin, possibly dating 3.5-3.8 Ga ago, than previously assumed (Marzari et al. 1996).

For the NEOs, given their short evolution timescale in near-Earth space $(\sim 5 \mathrm{Myr})$, the major contribution to their space weathering alteration comes from their past evolution into the main belt. Therefore, for each NEO we set $a$ equal to its computed average source distance (Marchi et al. $2006 \mathrm{a}$ ) and $e=0.17$ (the average of MBAs).

\section{Space weathering on V-types?}

The main results of our model can be investigated with the help of the slope-exposure scatter plot. For this purpose, we separated V-types belonging to the Vesta dynamical family from non-family members (Roig \& Gil-Hutton 2006). Results are shown in Figure 1 For comparison, the trend derived for S-types is also shown. Note that the latter is computed by the least-square 
fitting of S-type visible spectra of MBAs detected by SMASS and S3OS2 surveys (a total of 750 objects), using the SDSS bands fitting procedure, as described above. This trend is very close to the one derived by means of spectroscopic data in Paolicchi et al. (2007) (valid for $E>10$ ), ensuring that the procedure adopted here is robust. Figure 1 also reports V-type NEOs along with average slopes and range of slopes for a sample of 50 HEDs. Note that these HED samples have similar grain size $(<25 \mu \mathrm{m})$; thus, the observed spread in slope is likely due to differences in composition, rather than due to grain size effects.

A number of interesting results can be derived from Figure 1. First of all, V-types are, on average, redder than HEDs. This difference is minimized for eucrite meteorites. To better study this difference, we report the slope histograms in Figure2. The average slope gap between eucrites and MBA V-types is about $0.6 \mu \mathrm{m}^{-1}$, similar to that of S-types and OCs (Paolicchi et al. 2007). The largest difference, of about $1.0 \mu \mathrm{m}^{-1}$, is found for diogenites and MBA V-types.

It is not easy to understand the origin for this marked difference with available data. It could be due, for instance, to systematic differences in grain size or observational conditions, such as the phase angle. However, it is hard to believe that these factors can be entirely responsible for the observed slope variations for the following reasons. Concerning the grain size, we computed the effect of the particle size on the slope by analyzing two eucrites and one diogenite for which spectra with different particle sizes (in the range 25-250 $\mu \mathrm{m}$ ) have been gathered (RELAB database). In this range, the slope increases with decreasing particle sizes by about $0.4 \mu \mathrm{m}^{-1}$ (see also Hiroi \& Pieters 1998; Burbine et al. 2001). Therefore, to obtain the observed reddening on asteroids, their particle size should be much smaller than $25 \mu \mathrm{m}$, a conclusion which seems unrealistic given the lowgravity environment of V-types 1 . Moreover, we recall that the HED slopes used here are virtually the reddest, given that the particle size of these samples is below $25 \mu \mathrm{m}$. Concerning the phase

\footnotetext{
${ }^{1}$ For comparison, the average regolith particle size on the Moon is $70 \mu \mathrm{m}$, and only $10-20 \%$ of the particles have sizes $<20 \mu \mathrm{m}$. Moreover, thermal inertia modeling shows that asteroid's regolith is much coarser than the lunar one (e.g. Delbo' \& Tanga 2009).
}

angle of observations, it spans from about 2 to $30 \mathrm{deg}$, which is not too different from geometry conditions during spectra acquisition of HED samples (Burbine et al. 2001). There also seem to be differences between family and non-family V-types. The latter shows a larger slope scatter and has a considerable number of objects having slope $<-0.6 \mu \mathrm{m}^{-1}$ and $>0.5 \mu \mathrm{m}^{-1}$ (see Fig. 2). These two extreme classes of object are separated by the bulk of the slope distribution, and might represent two distinct populations of objects. The "blue" non-family V-types $\left(<-0.6 \mu \mathrm{m}^{-1}\right)$ are compatible with HEDs, and do not show any effect of spectral alteration.

The "red" V-types $\left(>0.5 \mu \mathrm{m}^{-1}\right)$, are much redder than HEDs. Their exposure is not systematically different than other V-types; therefore the observed red slope is not justifiable in terms of space weathering. Possible interpretations are that: either i) they are S-types that are erroneously classified as V-types or ii) if basaltic, they have a peculiar composition which is not sampled by HEDs. After a close look at SDSS data for these objects, it seems that i) could be ruled out; however, v-nir spectroscopy is needed for a secure classification (see end of this section for further comments on this issue). Moreover, these extreme slope objects do not have any significant difference in terms of orbital parameters with respect to other V-types. Although less evident, blue and red objects are also present among family $\mathrm{V}$-types.

Interestingly, NEO V-types are somehow in between HEDs and MBAs. This is, at least for some NEOs, in agreement with their computed intermediate exposure, as also found for S-types (Paolicchi et al. 2007). Nevertheless, the optical properties of NEOs can be affected by other processes like close encounters (Marchi et al. 2006b; Binzel et al. 2010). The effects of the latter on Vtype NEOs will be investigated in a future work. Alternatively, several differences with S-types can be noted. The most striking is that the slope shows a negative trend for increasing exposures. In other words, the more exposed objects tend to appear bluer, on average, than less exposed ones. The trend is less pronounced for non-family members, and becomes remarkably noticeable for family members. A linear best fit performed through minimizing chi-squared error statistics gives the following trends (expressed in units of 
slope $\left.\left[\mu \mathrm{m}^{-1}\right] / \log \left(E\left[\mathrm{MaAU}^{-2}\right]\right)\right):-0.71 \pm 0.02$ and $-0.35 \pm 0.02$ for family and non-family V-types, respectively. Error bars are estimated taking into account 1-sigma errors of slope values. Both trends are statistically highly significant with a tdistribution two-tailed probability of significance of $10^{-6}$ and $10^{-9}$, respectively 2 . For comparison, the S-types trend is $0.23 \pm 0.07$ (two-tailed probability of $10^{-3}$ ).

In light of the above results, the unexplained lack of spectral reddening on Vesta can be studied from a new perspective. First of all, there seems to be a general de-reddening trend with increasing exposures. These trends are also marginally present in terms of diameters, and become progressively more pronounced in terms of ages and exposures. This result suggests that the trends are not merely due to an asteroid size effect. Note that Vesta being merely the largest body (i.e. the most exposed) fits with this trend.

The slope scatter of V-types is larger than that of HEDs, however the latter may be limited due to the relatively low number of samples used in this work. Therefore, as for the HEDs, it is possible that much of the V-type slope spread may be due to compositional/texture differences. It could also be (partially) due to the presence of S-type -erroneously classified as V-types- interlopers within the lists used in this work. S-types are expected to have, on average, higher slopes than V-types (Bus \& Binzel 2002). Therefore, the possible presence of S-type interlopers may affect the observed excess of red objects, which likely has a negligible influence on the clear slope gap between HED and candidate V-types shown in Fig. 2

\section{Discussion and Conclusion}

The main result of this paper is that V-type slopes are systematically redder than HEDs (see Fig. 1) Fig. 2). Thanks to a large sample of candidate V-types, the present work considerably extends previous results (e.g. Hiroi \& Pieters 1998; Burbine et al. 2001) and allows quantitative estimates of the reddening of V-types. The spectral mismatch mimics what was found for OCs and S-types (Paolicchi et al. 2007). The observed dis-

\footnotetext{
${ }^{2} \mathrm{~A}$ two-tailed probability less than $10^{-2}$ indicates full statistical significance of the correlation.
}

crepancy could be due to factors such as systematic compositional/texture differences, different conditions of observations or space weathering. We showed that observational conditions and texture are unlikely to be the source of the observed discrepancy. Therefore, either the marked slope shift between HEDs and V-types is due to systematical differences in composition, or it can be ascribed to some kind of space weathering alteration. The previous hypothesis would imply that the suite of HED compositions is not representative of the whole V-type population. While this is certainly an open possibility, it is mandatory to obtain v-nir spectra of small V-types to confirm. In the case that the slope mismatch is due to space weathering processes, it is possible to constrain the reddening timescale on the basis of the collisional ages. Although a precise estimate is not possible with present data, an upper limit for the reddening timescale is set by the youngest $\mathrm{V}$-type MBAs which have ages of $\sim 0.5$ Ga. Moreover, $58 \%$ of V-type NEOs have a slope compatible with HEDs (within 1-sigma of average values), while only about $20 \%$ of S-type NEOs are compatible with OCs (Binzel et al. 2010). Therefore, if no other effect comes into play, it can be inferred that V-type's space weathering timescale is larger than a factor of $\sim 3$ with respect to that of S-types.

The possibility that space weathering is responsible for the spectral mismatch is also reinforced by the few experiments published to date of ion bombardment of Bereba eucrite and Johnstown diogenite Hiroi \& Pieters 1998; Vernazza et al. 2006). They both exhibit reddening (and darkening). Note that these experiments also confirm a slower timescale with respect to OCs.

The true nature of the spectral mismatch still remains elusive in several aspects; nevertheless some noteworthy characteristics have been identified. In particular, we explore in detail the possibility that the spectral mismatch is due to space weathering processes.

First of all, the spectral slope anti-correlates with exposure to solar wind. This is the opposite of what was found for S-types and other spectral types (Lazzarin et al. 2006). Therefore, if some kind of Sun-related space weathering is operating on V-types, either it behaves in a different manner with respect to S-types or other factors wipe out 
the reddening-exposure relation. Concerning the latter, a possibility is represented by a composition gradient across V-types, from eucrite-like for the smallest members $(\sim 1 \mathrm{~km})$ to diogenite-like for the largest members $(\sim 10 \mathrm{~km})$. This putative composition gradient would help to explain the observed slope-exposure trend for two reasons: i) diogenites are bluer, on average, than eucrites; and ii) according to the few experiments available, the reddening of diogenites seem less pronounced than for eucrites, although the space parameters of HED compositions and alteration processes are far from being exhaustively investigated.

Note that the above explanation is preferred with respect to other possibilities, e.g. grain size variation, since the latter would require a grain size finer than $\sim 25 \mu \mathrm{m}$ (i.e. higher slope) for smaller objects. This is the opposite of what is expected, since smaller objects tend to have a larger grain size due to the low gravity. Also, the substantial presence of S-type interlopers among the red Vtypes would not greatly affect the observed slopeexposure trend since they are nearly equally distributed in terms of $E$ (see Fig. 1).

If confirmed, the above compositional trend among family V-types could be the result of cratering ejecta from various depths in Vesta's crust (eucrite is expected in the upper crust, while diogenite is expected in the lower crust); or may be due to different episodes of cratering occurring in regions with different compositions. However, since large fragments are expected to originate in the near-surface spall region, the second hypothesis seems more probable. This may also be confirmed by the variety ${ }^{39} \mathrm{Ar}-{ }^{40} \mathrm{Ar}$ shock ages of HEDs (Bogard \& Garrison 2003). Furthermore, the spectral data of $\mathrm{V}$-types seem to indicate that both mineralogies are present (Duffard et al. 2004), but the limited sample prevents a firm conclusion.

The proposed scenario could be tested as soon as more v-nir spectra of small V-types would become available. Note that the above discussion holds for both family and non-family V-types. For the latter, however, the anti-correlation is less pronounced, and it becomes nearly flat if the outliers (red and blue) are excluded. This could be an indication that most non-family $\mathrm{V}$-types have a different origin with respect to family V-types.

The NASA Dawn mission will aid in understand- ing the weathering process on Vesta, thanks to spectral imaging and global mapping. Therefore, the possibility to have detailed spectral information of specific areas of different composition and texture will shed light on the different effects of space weathering on basaltic asteroids.

We thank the anonymous referee for the helpful comments on the manuscript.

\section{REFERENCES}

Benner, L. A. M., et al. 2002, Icarus, 158, 379

Binzel, R. P., \& Xu, S. 1993, Science, 260, 186

Binzel, R. P., Rivkin, A. S., Stuart, J. S., Harris, A. W., Bus, S. J., \& Burbine, T. H. 2004, Icarus, 170, 259

Binzel, R. P., et al. 2010, Nature, 463, 331

Bogard, D. D., \& Garrison, D. H. 2003, Meteoritics and Planetary Science, 38, 669

Bottke, W. F., Durda, D. D., Nesvorný, D., Jedicke, R., Morbidelli, A., Vokrouhlický, D., \& Levison, H. 2005, Icarus, 175, 111

Burbine, T. H., Buchanan, P. C., Binzel, R. P., Bus, S. J., Hiroi, T., Hinrichs, J. L., Meibom, A., \& McCoy, T. J. 2001, Meteoritics and Planetary Science, 36, 761

Bus, S. J., \& Binzel, R. P. 2002, Icarus, 158, 146

Consolmagno, G. J., \& Drake, M. J. 1977, Geochim. Cosmochim. Acta, 41, 1271

Delbó, M., Harris, A. W., Binzel, R. P., Pravec, P., \& Davies, J. K. 2003, Icarus, 166, 116

Delbó, M., et al. 2006, Icarus, 181, 618

Delbo', M., \& Tanga, P. 2009, Planet. Space Sci., 57,259

Drake, M. J. 2001, Meteoritics and Planetary Science, 36, 501

Duffard, R., Lazzaro, D., Licandro, J., de Sanctis, M. C., Capria, M. T., \& Carvano, J. M. 2004, Icarus, 171,120

Duffard, R., \& Roig, F. 2009, Planet. Space Sci., 57,229 
Gaffey, M. J. 1997, Icarus, 127, 130

Hardersen, P. S., Gaffey, M. J., \& Abell, P. A. 2004, Icarus, 167, 170

Hiroi, T., \& Pieters, C. M. 1998, Antarctic Meteorite Research, 11, 163

Ivezić, Ž., et al. 2001, AJ, 122, 2749

Jedicke, R., Nesvorný, D., Whiteley, R., Ivezić, Ž., \& Jurić, M. 2004, Nature, 429, 275

Jurić, M., et al. 2002, AJ, 124, 1776

Larson, H. P., \& Fink, U. 1975, Icarus, 26, 420

Lazzarin, M., Marchi, S., Moroz, L. V., Brunetto, R., Magrin, S., Paolicchi, P., \& Strazzulla, G. 2006, ApJ, 647, L179

Lazzaro, D., et al. 2000, Science, 288, 2033

Lazzaro, D., Angeli, C. A., Carvano, J. M., MothéDiniz, T., Duffard, R., \& Florczak, M. 2004, Icarus, 172,179

Marchi, S., Brunetto, R., Magrin, S., Lazzarin, M., \& Gandolfi, D. 2005a, A\&A, 443, 769

Marchi, S., Lazzarin, M., Paolicchi, P., \& Magrin, S. 2005b, Icarus, 175,170

Marchi, S., Paolicchi, P., Lazzarin, M., \& Magrin, S. 2006a, AJ, 131, 1138

Marchi, S., Magrin, S., Nesvorný, D., Paolicchi, P., \& Lazzarin, M. 2006b, MNRAS, 368, L39

Marzari, F., Cellino, A., Davis, D. R., Farinella, P., Zappala, V., \& Vanzani, V. 1996, A\&A, 316, 248

McCord, T. B., Adams, J. B., \& Johnson, T. V. 1970, Science, 168, 1445

Nesvorný, D., Roig, F., Gladman, B., Lazzaro, D., Carruba, V., \& Mothé-Diniz, T. 2008, Icarus, 193,85

Paolicchi, P., Marchi, S., Nesvorný, D., Magrin, S., \& Lazzarin, M. 2007, A\&A, 464, 1139

Pieters, C. M., Binzel, R. P., Bogard, D., Hiroi, T., Mittlefehldt, D. W., Nyquist, L., Rivkin, A., \& Takeda, H. 2006, Asteroids, Comets, Meteors, 229, 273
Russell, C. T., et al. 2007, Earth Moon and Planets, 101,65

Roig, F., \& Gil-Hutton, R. 2006, Icarus, 183, 411

Roig, F., Nesvorný, D., Gil-Hutton, R., \& Lazzaro, D. 2008, Icarus, 194, 125

Takeda, H. 1997, Meteoritics and Planetary Science, 32,841

Tedesco, E. F., Noah, P. V., Noah, M., \& Price, S. D. 2002, AJ, 123, 1056

Thomas, P. C., Binzel, R. P., Gaffey, M. J., Storrs, A. D., Wells, E. N., \& Zellner, B. H. 1997, Science, 277,1492

Vernazza, P., Brunetto, R., Strazzulla, G., Fulchignoni, M., Rochette, P., Meyer-Vernet, N., \& Zouganelis, I. 2006, A\&A, 451, L43

Wiechert, U. H., Halliday, A. N., Palme, H., \& Rumble, D. 2004, Earth and Planetary Science Letters, 221, 373

Yamaguchi, A., et al. 2002, Science, 296, 334

This 2-column preprint was prepared with the AAS LATEX macros v5.2. 


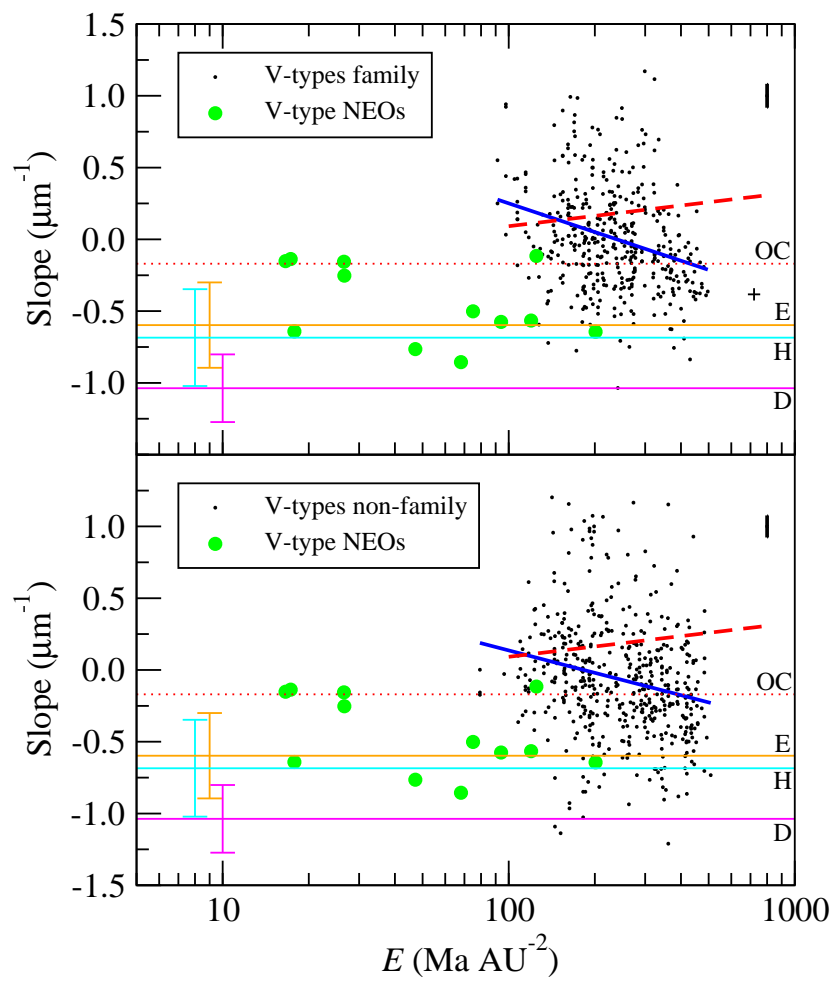

Fig. 1.- Slope vs exposure scatter plot for MBA V-types. Both family and non-family V-types are reported. The position of Vesta is indicated by the plus sign (upper panel). Horizontal lines indicate the average values for a sample of 14 howardites, 26 eucrites, 10 diogenites and 180 OCs. The range of slope values for HEDs (computed as 1-sigma of the slopes) is also reported (placed arbitrarily at $E=8,9,10$ ). NEOs slopes are indicated by filled green circles. Red dashed line indicates the reddening trend for S-types, while blue lines indicate MBA V-types best fit. The average 1-sigma slope error is reported at the upper-right corner of both panels. 


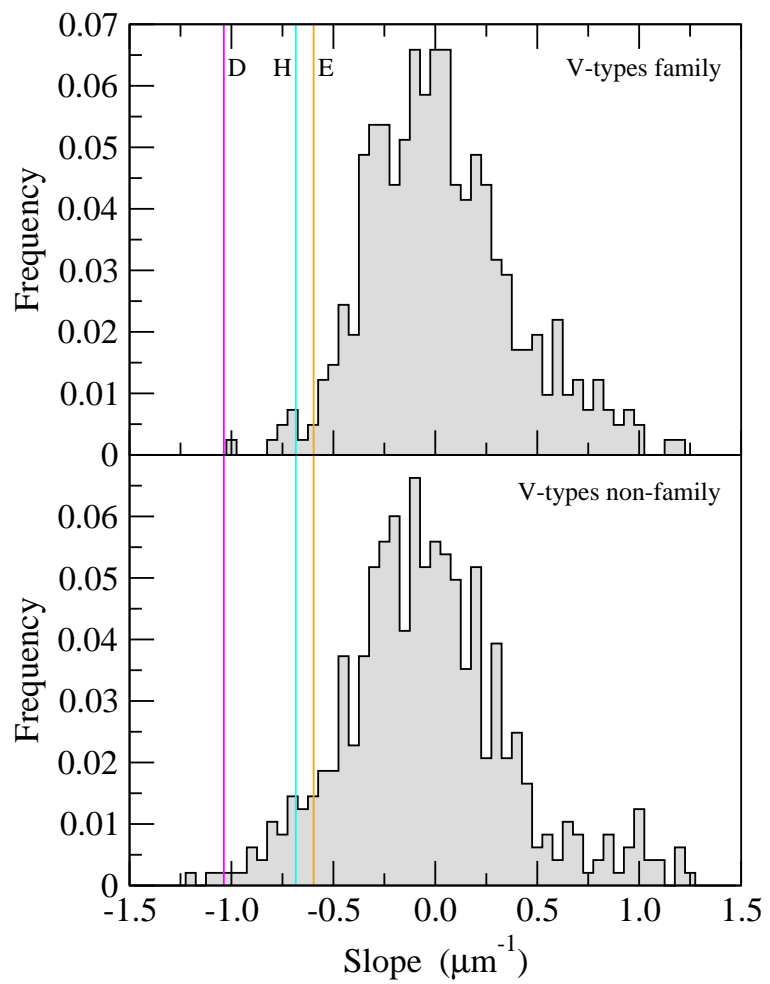

Fig. 2.- Histograms of visible slopes for family and non-family V-types. Average slope values of HEDs are also indicated by vertical lines (see also Figure 11). Note that both distributions show marked non-Gaussian shapes, with a clear excess of "blue" and "red" objects, i.e. having slopes $<-0.6 \mu \mathrm{m}^{-1}$ and $>0.5 \mu \mathrm{m}^{-1}$, respectively (see the text for details). 